\title{
SAMPEX MISSION OVERVIEW
}

\author{
G. M. Mason ${ }^{a}$, D. N. Baker ${ }^{b}$, J. B. Blake ${ }^{c}$, L. B. Callis ${ }^{d}$,
}

D. C. Hamilton ${ }^{a}$, D. Hovestadt ${ }^{e}$, B. Klecker ${ }^{e}$, R. A. Mewaldt $f$, M. Scholer ${ }^{e}$, E. C. Stone ${ }^{f}$, and T. T. von Rosenvinge ${ }^{b}$

${ }^{a}$ University of Maryland, College Park, MD 20742

${ }^{b}$ NASA/Goddard Space Flight Center, Greenbelt, MD 20771

${ }^{c}$ Aerospace Corporation, Los Angeles, CA 90009

${ }^{d}$ NASA/Langley Research Center, Hampton, VA 23665-5225

${ }^{e}$ Max-Planck-Institut für extraterrestrische Physik, 8046 Garching, FRG

${ }^{f}$ California Institute of Technology, Pasadena, CA 91125

\section{ABSTRACT}

The Solar, Anomalous, and Magnetospheric Particle Explorer, SAMPEX, will carry out energetic particle studies of outstanding scientific questions in the fields of space plasma physics, solar physics, magnetospheric and middle atmospheric physics, and cosmic ray physics. SAMPEX will measure the electron and ion composition of energetic particle populations from $\sim 0.4 \mathrm{MeV} /$ nucleon to hundreds of $\mathrm{MeV} /$ nucleon from a zenith-pointing small satellite in near-polar orbit, using a coordinated set of detectors with excellent charge and mass resolution, and with higher sensitivity than previously flown instruments. While over the magnetic poles, the instruments will study the composition of anomalous cosmic rays, solar energetic particles, and galactic cosmic rays. At lower magnetic latitudes, geomagnetic cutoff effects will allow determination of the ionization state of these particles at energies much higher than can be studied from interplanetary spacecraft. At subauroral latitudes, SAMPEX will also observe precipitating relativistic magnetospheric electrons, which undergo important interactions within the middle atmosphere.

\section{ANOMALOUS COSMIC RAYS}

A major goal of the SAMPEX mission is to determine the ionization state of the Anomalous Cosmic Rays (ACRs), elements ( $\mathrm{He}, \mathrm{C}, \mathrm{N}, \mathrm{O}, \mathrm{Ne}$, and $\mathrm{Ar}$ ) in the energy range below $\sim 50 \mathrm{MeV}$ /nucleon whose abundances are anomalous compared to other solar system material. For example the oxygen;carbon ratio in ACRs is $>50$, while in the 'universal abundances' this ratio is close to 2 . A widely accepted model for ACRs makes a unique prediction: ACRs should be singly ionized. SAMPEX will give the first definitive measurements of the charge state of anomalous $\mathrm{He}$ and $\mathrm{O}$, assuming that their flux during 1993 and 1994 has recovered as expected from low solar maximum levels.

In the case of ACR oxygen, when SAMPEX is over the geomagnetic poles it will measure the interplanetary flux levels. If this oxygen is fully stripped the 
geomagnetic field will prevent it from reaching the satellite when it is more than $\sim 25^{\circ}$ from the magnetic poles; if it is singly ionized, it will reach the spacecraft up to $\sim 40^{\circ}$ from the poles - a simple measurement. Even though ACR fluxes are low, the sensitive detectors on SAMPEX will collect thousands of counts/yearmore than enough to discriminate between singly and fully ionized oxygen charge states.

If the ACR component is shown to be singly charged, then this component represents a direct sample of the local interstellar medium that carries important information about galactic evolution in the solar neighborhood over the time interval since the formation of the solar nebula; SAMPEX will measure the isotopic composition of this sample of matter.

\section{PRECIPITATING RELATIVISTIC MAGNETOSPHERIC ELECTRONS}

Another unique study possible with the combination of SAMPEX instruments and orbit concerns precipitating relativistic magnetospheric electrons. Observations at synchronous orbit have found $\gtrsim 1 \mathrm{MeV}$ electron intensities that increase substantially for periods of a few days often with a recurrence period of $\sim 27$ days. Numerical models show that the multi-MeV electrons-when they precipitate-can cause a large $\left(10-100 \mathrm{keV} / \mathrm{cm}^{3}-\mathrm{s}\right)$ energy deposition at $40-60$ $\mathrm{km}$ altitude in the atmosphere. This could dominate other ionization sources (such as cosmic rays and solar EUV) at these heights by orders of magnitude.

Precipitating relativistic electrons may indeed lead to substantial long-term increases in the levels of odd nitrogen compounds $\left(N \mathrm{O}_{x}\right)$ at these heights with an attendant impact on local ozone levels via the reactions $\mathrm{NO}+\mathrm{O}_{3} \rightarrow \mathrm{NO}_{2}+\mathrm{O}_{2}$, and $\mathrm{NO}_{2}+\mathrm{O} \rightarrow \mathrm{NO}+\mathrm{O}_{2}$. It is therefore a critical problem to determine the actual intensity and spatial extent of relativistic electron precipitation. SAMPEX will determine the longitude, latitude, and local time precipitation characteristics for $>1 \mathrm{MeV}$ electrons required for these studies.

\section{SOLAR ENERGETIC PARTICLES}

By launching into the period shortly after sunspot maximum, SAMPEX should observe several large solar particle events; as the solar cycle winds down, it will observe numerous implusive flares such as ${ }^{3} \mathrm{He}$-rich flares. Solar energetic particles provide a sample of ions from the solar atmosphere. Since the charge states of SEPs are determined by the plasma conditions at the acceleration site, measurements of the average charge state can be used as a diagnostic tool. For example, previous limited measurements at low energies have already revealed large ( $\sim 40 \%$ for $\mathrm{Fe})$ differences between the charge states of $\mathrm{Si}$ and $\mathrm{Fe}$ in ${ }^{3} \mathrm{He}$ rich events and those in events with normal composition, indicating that the ${ }^{3}$ He-rich flares originate in hot sites in the corona. Using geomagnetic cutoff techniques, SAMPEX will measure the mean charge states of these solar flare elements with greater statistical accuracy, and to significantly higher energies than previous missions. 
The solar energetic particle composition provides a direct measure of the present solar elemental and isotopic makeup, providing crucial information for understanding the history of solar system material and adding new dimensions to the study of solar flare acceleration and propagation processes. It is almost a decade since the first high-resolution measurements of solar flare isotopes heavier than He were provided by an instrument on ISEE-3. Since that time little progress has been made because no instruments with improved collecting power have been launched, and this field has therefore remained relatively unexplored. Spectrometers on SAMPEX will resume these studies and will significantly improve on the collecting power of past instruments.

\section{GALACTIC COSMIC RAYS}

A final area of the SAMPEX scientific studies involves galactic cosmic rays, which are a directly accessible sample of matter from outside the solar system. A spectrometer on SAMPEX will carry out measurements of the isotopic composition of this sample of high energy matter, which contains a record of the nuclear history of cosmic rays including their synthesis in stars and subsequent nuclear interactions with the interstellar gas. Cosmic ray isotope observations have already revolutionized our views of both cosmic ray origin and propagation. As an example, measurements by a number of groups have shown that ${ }^{22} \mathrm{Ne}$ is a factor of at least 3 times more abundant in cosmic ray source material than in the solar system, while the abundances of ${ }^{25} \mathrm{Mg},{ }^{26} \mathrm{Mg},{ }^{29} \mathrm{Si}$, and ${ }^{30} \mathrm{Si}$ are all enhanced by a factor of $\sim 1.5$. The exposure obtained on SAMPEX will make it possible to extend the search for isotopic differences between galactic and solar cosmic ray material to several additional key elements.

Table 1. Instrument Performance

LEICA HILT MAST PET

$\begin{array}{lllll}\begin{array}{l}\text { Energy range for } \\ \text { electrons }\end{array} & - & - & - & 1-120 \mathrm{MeV} \\ \mathrm{H} & 0.75-6 & - & 7-15 & 18-350 \mathrm{MeV} \\ \mathrm{He} & 0.4-6 & 3.9-90 & 7-91 & 18-500 \mathrm{MeV} / \mathrm{n} \\ \mathrm{C} & 0.35-12 & 7.2-160 & 12-210 & \\ \mathrm{Si} & 0.26-6 & 9.6-177 & 19-345 & 54-195 \mathrm{MeV} / \mathrm{n} \\ \mathrm{Fe} & 0.16-4 & 11.0-90 & 24-470 & \\ \text { Charge range for elements } & 1-28 & 2-28 & 1-28 & 1-28 \\ \text { Charge range for isotopes } & 2-16 & 2 & 1-28 & 1-2 \\ \left.\text { Geometrical factor (cm }{ }^{2} \mathrm{sr}\right) & 1.0 & 50 & 7-16 & 0.3-1.6\end{array}$




\section{INSTRUMENTATION AND MISSION DESCRIPTION}

The SAMPEX instrumentation includes a complementary set of four high resolution, high sensitivity particle detectors: the Heavy Ion Large Telescope (HILT), the Low Energy Ion Composition Analyzer (LEICA), the Mass Spectrometer Telescope (MAST), and the Proton/Electron Telescope (PET). Prototypes of LEICA and HILT flew on the Space Shuttle in August, 1989. MAST and PET were originally approved and under construction for the (cancelled) U.S. spacecraft of the International Solar Polar Mission. Thus, these new instruments are in an advanced state of development and can be readied for SAMPEX quickly and at moderate cost. Table 1 lists instrument characteristics, and Table 2 gives mission and spacecraft parameters.

The broad range of studies carried out by SAMPEX could only be carried out on a low altitude near-polar orbiting spacecraft such as can be flown under the Small-Class Explorer Program. The 1992 launch date will allow important solar studies to be carried out during a period of enhanced solar activity and continuing into the declining phase of the activity cycle. This time interval is not only ideal for the objectives discussed above but also would provide coverage of energetic particle fluxes in the period preceding the launch of the WIND spacecraft. SAMPEX will also provide key particle data to complement the measurements of Solar-A, GOES, and GRO during the solar maximum, as well as provide baseline coverage for Ulysses, Galileo, and the Pioneer and Voyager spacecraft. SAMPEX will also provide overlapping coverage for a number of long duration balloon flights planned as part of the Max '91 program. SAMPEX precipitating particle measurements will be compared with observations on geostationary satellites and CRRES, and also with ozone measurements from UARS.

Table 2. SAMPEX Mission Description

Orbit: $580 \mathrm{~km}$ circular, $\mathbf{8 2 ^ { \circ }}$ inclination Launch Date: first half 1992

Mass: $58 \mathrm{~kg}$ instruments, $119 \mathrm{~kg} \mathrm{~S} / \mathrm{C}+$ margin Power: $20 \mathrm{~W}$ instruments, $55 \mathrm{~W} \mathrm{~S} / \mathrm{C}$

Spacecraft: to be built at GSFC
Orbit average bitrate: $3 \mathrm{kpbs}$ Mission Duration: $\geq 3$ years Total: $177 \mathrm{~kg}$ Total: $75 \mathrm{~W}$

Launch site: Vandenberg AFB

Acknowledgements: This work is supported in part by NASA contract NAS5-30704 and by the German Government. 